\title{
Análise da influência da homozigose dos alelos HLA no tempo de espera para realização do transplante renal no Ceará
}

\author{
Analysis of the influence of homozygosis of alleles HLA on the waiting time for kidney \\ transplantation
}

Análisis de la influencia de la homocigosis de alelos HLA en el tiempo de espera para trasplante renal en Ceará

Karen Moanny Pereira de Sousa ORCID: https://orcid.org/0000-0001-8537-9563 Universidade de Fortaleza, Brasil E-mail: karenmoanny@edu.unifor.br

Nathália Bezerra Barbosa

ORCID: https://orcid.org/0000-0003-3295-5242

Universidade de Fortaleza, Brasil

E-mail: nati.bezerra15@gmail.com

Ilana Farias Ribeiro Araujo

ORCID: https://orcid.org/0000-0001-5470-2479 Centro de Pesquisa em Doenças Hepato Renais do Ceará, Brasil E-mail: ilanafribeiro@hotmail.com

Larissa Cavalcante de Freitas

ORCID: https://orcid.org/0000-0003-0875-4758 Centro de Pesquisa em Doenças Hepato Renais do Ceará, Brasil

E-mail: lariscavalcante@ hotmail.com

Mauriclécio Franco Ponte

ORCID: https://orcid.org/0000-0001-7935-575X Centro de Pesquisa em Doenças Hepato Renais do Ceará, Brasil E-mail: mf.ponte@hotmail.com

Paulo Goberlânio de Barros Silva ORCID: https://orcid.org/0000-0002-1513-9027 Centro Universitário Christus, Brasil

E-mail: paulo_goberlanio@yahoo.com.br

Eliana Régia Barbosa de Almeida ORCID: https://orcid.org/0000-0002-8359-4099

Central de Transplantes do Ceará, Brasil E-mail: elianaregia@gmail.com

Sônia Leite da Silva

ORCID: https://orcid.org/0000-0003-2732-8543 Universidade Federal do Ceará, Brasil Universidade de Fortaleza, Brasil E-mail: sonials@unifor.br

Silvia Fernandes Ribeiro da Silva ORCID: https://orcid.org/0000-0002-3223-5184 Centro Universitário Christus, Brasil Universidade de Fortaleza, Brasil E-mail: silviafernandes@unifor.br

\begin{abstract}
Resumo
O objetivo do estudo foi avaliar se a homozigose dos alelos do Sistema HLA influenciou o tempo de espera para realização do transplante renal no Ceará. Trata-se de um estudo transversal realizado a partir da busca ativa de prontuários de pacientes que transplantaram com rim de doador falecido no estado do Ceará, entre janeiro de 2018 a dezembro de 2019. A análise dos dados foi realizada com 406 pacientes, 84 foram excluídos. A média de idade e o tempo de espera foram de $47 \pm 13,1$ anos e 14,64 $\pm 16,18$ meses, respectivamente. A maioria era do sexo masculino $(62,3 \%)$ com média de idade superior à do sexo feminino $(\mathrm{p}=0,0034)$. A média do tempo de espera foi maior no grupo de pacientes que tinha entre 36 e 45 anos $(\mathrm{p}=0,002)$ e naqueles do grupo $\mathrm{O}$ e grupo $\mathrm{A}(\mathrm{p}=0,001)$. Não foi observada influência da homozigose $(p=0,934)$, do número de homozigoses $(p=0,448)$ e da presença de homozigoses nos diferentes loci HLA (locus A, p=0,169, locus B, p=0,629 e locus DR, p=0,064) sobre o tempo de espera. A homozigose dos alelos do sistema HLA não influenciou no tempo de espera na lista para transplante renal no Ceará no período avaliado, mesmo em pacientes com PRA elevado. Estudos são necessários para melhor avaliar a influência da
\end{abstract}


homozigose HLA em um número maior de receptores, em especial no locus DR. Esses resultados serão importantes para se rever os critérios de seleção dos potenciais receptores de rim, uma vez que atualmente a homozigose não é pontuada.

Palavras-chave: Histocompatibilidade; Transplante de rim; Homozigose; Lista de espera.

\begin{abstract}
The aim of the study was to evaluate whether the homozygosity of the HLA System alleles presented by transplant recipients in Ceará influenced the waiting time for kidney transplantation. This is a cross-sectional study, carried out based on the active search for medical records, of patients who transplanted with a kidney from a deceased donor in the state of Ceará, from January 2018 to December 2019. Data analysis was performed with 406 patients, 84 were excluded. The mean age and waiting time of $47 \pm 13.1$ years and $14.64 \pm 16.18$ months, respectively, were observed. Most patients were male $(62.3 \%)$ with a mean age greater than women $(p=0.0034)$. The average waiting time was higher in the group of patients who were between 36 and 45 years old $(p=0.002)$ when compared to the other age groups, while the waiting time was higher in group $\mathrm{O}$ and group $\mathrm{A}(\mathrm{p}=0.001)$. There was no influence of homozygosity $(p=0.934)$, number of homozygosis $(p=0.448)$ and presence of homozygosis in different HLA loci (locus A, p=0.169, locus B, p=0.629 and locus DR, p=0.064) on the waiting time. Thus, homozygosity in the different alleles of the HLA system did not influence the waiting time on the list of patients who transplanted in 2018 and 2019. Studies are necessary to better assess the influence of HLA homozygosity in a larger number of receptors, especially in the DR locus. These results will be important to review the selection criteria for potential kidney receptors, since homozygosis is currently not scored.
\end{abstract}

Keywords: Histocompatibility; Kidney transplantation; Homozygosis; Waiting list.

\title{
Resumen
}

El objetivo del estudio fue evaluar si la homocigosidad de los alelos del sistema HLA influenció en el tiempo de espera para el trasplante renal en Ceará. Se trata de un estudio transversal realizado a partir de la búsqueda activa de historias clínicas de pacientes trasplantados con riñón de donante fallecido en el estado de Ceará, entre enero de 2018 y diciembre de 2019. El análisis de datos se realizó con 406 pacientes, 84 fueron excluidos. La edad media y el tiempo de espera fueron $47 \pm 13,1$ años y 14,64 $\pm 16,18$ meses, respectivamente. La mayoría eran hombres $(62,3 \%)$ con una edad media superior a la de las mujeres $(\mathrm{p}=0,0034)$. El tiempo medio de espera fue mayor en el grupo de pacientes entre 36 y 45 años $(\mathrm{p}=0,002)$ y en los del grupo $\mathrm{O}$ y el grupo $\mathrm{A}(\mathrm{p}=0,001)$. No hubo influencia de la homocigosis $(\mathrm{p}=0,934)$, del número de hemocigosis $(\mathrm{p}=0,448)$ y de la presencia de homocigosis en los diferentes loci HLA (locus $\mathrm{A}, \mathrm{p}=0,169$, locus $\mathrm{B}, \mathrm{p}=0,629$ y locus $\mathrm{DR}, \mathrm{p}=0,064)$ sobre el tiempo de espera. La homocigosidad de los alelos del sistema HLA no influenció en el tiempo de espera en la lista de trasplante de riñón en Ceará para los pacientes trasplantados durante el período evaluado, incluso en pacientes con PRA elevado. Se necesitan estudios para evaluar la influencia de la homocigosis en un mayor número de receptores, especialmente en el locus DR. Estos resultados serán importantes para se revisar los criterios de selección de los posibles receptores renales, ya que actualmente no se puntúa la homocigosidad.

Palabras clave: Histocompatibilidad; Trasplante de riñón; Homocigosis; Lista de espera.

\section{Introdução}

A Doença Renal Crônica (DRC) é considerada um problema de saúde pública mundial, afetando mais de 750 milhões de pessoas em todo o mundo (Crews, et al., 2019). No Brasil, o número exato de pessoas com DRC ainda é incerto; contudo, estima-se que 3 a 6 milhões de adultos podem apresentar esta doença (Romão, 2004). O risco de desenvolver DRC é maior em indivíduos com idade avançada, diabéticos, hipertensos, obesos, tabagistas, que fazem uso crônico de drogas nefrotóxicas, com histórico familiar de DRC e/ou doença cardiovascular (Marinho, et al., 2017).

As principais formas de tratamento da DRC são a diálise e o transplante renal (Brasil, 2014). Porém, atualmente, o transplante renal (TxR) é considerado o melhor tratamento, pois tem maior sobrevida do paciente e melhor qualidade de vida, além de representar menor custo do que o tratamento dialítico (Santos, et al., 2018). Segundo a Sociedade Brasileira de Nefrologia (SBN, 2019), o TxR consiste na substituição do rim doente por um órgão sadio e capaz de desempenhar plenamente as funções perdidas. Para tanto, é imperativo que o paciente seja encaminhado para um serviço de TxR, que avaliará se o paciente tem condições clínicas de ser submetido a este procedimento e a disponibilidade de doador vivo parente. Na ausência de doador aparentado, ele é inscrito na lista de espera da Central de Transplante (CETx) do seu estado para realizar o TxR com um rim proveniente de um doador falecido (Garcia, et al., 2015). 
Independentemente do tipo de doador, os exames de histocompatibilidades devem ser realizados no preparo da dupla receptor/doador para o TxR, como a tipagem sanguínea ABO, a tipagem HLA (Human Leukocyte Antigen), a prova cruzada e o PRA (Panel-Reactive Antibody) (Despande, 2017).

A tipagem HLA avalia a compatibilidade da dupla receptor/doador frente aos alelos de classe I (loci A e B) e de classe II (loci DR e DQ) do Sistema HLA. Esses alelos são codificados por genes do complexo principal de histocompatibilidade (MHC), localizados no braço curto do cromossoma 6 (Goldberg \& Rizzo, 2015; Despande, 2017). Além disso, são os antígenos de histocompatibilidade mais imunogênicos envolvidos na rejeição do TxR, sendo os alelos HLA DR o mais imunogênico, seguido dos alelos HLA B e HLA A (Santos, 2011). Vale ressaltar, que os loci A, B e DR são expressos de forma codominante, podendo totalizar de 0 a 6 incompatibilidades entre o receptor e o doador. A determinação das incompatibilidades HLA nos testes pré-transplante é importante, pois quanto maior a incompatibilidade, maior a probabilidade de rejeição e menor a sobrevida do enxerto (Delves, 2014; Despande, 2017).

A análise das incompatibilidades entre os alelos HLA da dupla receptor/doador exerce grande influência no somatório dos pontos necessários para seleção do receptor que se encontra na lista de espera das CETx. Em geral, a pontuação é realizada da seguinte forma: zero incompatibilidade no HLA DR recebe 10 pontos, no HLA B 4 pontos e no HLA A 1 ponto. Por outro lado, se este apresenta somente uma incompatibilidade, a pontuação atribuída para os loci DR, B e A é, respectivamente, $5,2 \mathrm{e}$ 0,5. Porém, se a dupla tiver duas incompatibilidades nos referidos loci, o receptor não recebe pontuação (Pecora, 2016). Assim, considere como exemplo dois receptores X e Y e observe os seus respectivos alelos HLA: a) alelos HLA A2, A33, B7, B8, DR1 e DR2 do receptor X; e, b) alelos HLA A2, A24, B7, B7, DR2, DR2 do receptor Y. Esses receptores têm a possibilidade de transplantar com um doador cuja tipagem HLA é A25, A33, B7, B15, DR2 e DR4. Diante desses resultados, o receptor $\mathrm{Y}$ deveria ter maior pontuação do que o receptor $\mathrm{X}$ devido à homozigose em $\mathrm{B}$ e DR com o doador. Porém, atualmente, esse fato não é observado, uma vez que não há pontuação diferenciada para a homozigose, ou seja, é pontuado somente uma compatibilidade na homozigose. Neste caso em questão o receptor $X$ teria maior pontuação (pontos= 7,5$)$ do que o Y (pontos= 7,0), por apresentar uma incompatibilidade no locus A com o doador.

Diante do exposto, é fato que a pontuação das incompatibilidades HLA A, B e DR representa um critério importante na seleção do receptor e a legislação vigente não pontua a homozigose. Assim, o objetivo do presente estudo foi avaliar se a homozigose dos alelos do Sistema HLA apresentada pelos receptores transplantados no Ceará no período de janeiro de 2018 a dezembro de 2019 influenciou no tempo de espera para realização do TxR.

\section{Metodologia}

Trata-se de um estudo observacional do tipo transversal realizado a partir da busca ativa de prontuários de pacientes com DRC que transplantaram com rim de doador falecido no estado do Ceará, no período de janeiro de 2018 a dezembro de 2019. Foram excluídos do estudo pacientes com idade inferior a 18 ou superior a 70 anos e aqueles que realizaram transplante duplo, ou seja, rim/pâncreas ou rim/fígado.

As seguintes variáveis dos pacientes transplantados foram avaliadas: sexo, idade, tipagem ABO, tipagem HLA, PRA, data de inscrição na lista de espera da CETx do Ceará e da realização do TxR. A idade dos pacientes foi distribuída em quatro intervalos: até 35 anos, de 36 a 45 anos, 46 a 55 anos e > 55 anos. Para análise da influência do PRA no tempo de espera, os resultados foram divididos em $0 \%, 1$ a $49 \%, 50$ a $79 \%$ e $\geq 80 \%$. O tempo que os pacientes ficaram na lista de espera para realização do TxR foi mensurado em meses.

Os dados obtidos foram organizados utilizando-se o programa Microsoft Office Excel $^{\circledR}$ versão 15.26, considerando-se as variáveis categóricas, expressas como frequência absoluta (n) e relativa (\%), e variáveis numéricas sendo apresentadas como média e desvio padrão. A análise do tempo de espera em lista com as variáveis categóricas e numéricas foi realizada 
utilizando-se o software Statistical Packcage for the Social Sciences (SPSS) versão 20,0 para Windows no qual as análises foram realizadas adotando uma confiança de $95 \%$. Foram calculadas as médias e desvio-padrão do tempo de espera em meses total e de cada variável avaliada, submetidos ao teste de normalidade de Kolmogorov-Smirnov e associadas utilizando o teste de Mann-Whitney ou Kruskal-Wallis/Dunn (dados não paramétricos). Ao final, o modelo de análise multivariada (regressão de Poisson) foi utilizado com todas as variáveis disponíveis para verificação de associações independentes.

O estudo atendeu as normas de ética em pesquisa envolvendo seres humanos - Registro CAAE - Certificado de Apresentação para Apreciação Ética: 38174620.0.000.5052.

\section{Resultados}

No período avaliado foram transplantados 480 pacientes no estado do Ceará, sendo 74 excluídos da amostra. Portanto, a análise dos dados foi realizada em 406 pacientes.

Como mostra a Tabela 1, a média de idade dos 406 pacientes foi de $47 \pm 13,1$ anos e a média do tempo de espera na lista da CETx do Ceará foi de 14,64 \pm 16,18 meses. A maioria dos pacientes era do sexo masculino $(62,3 \%)$, com média de idade superior à do sexo feminino $(48,5 \pm 12,8$ anos e 44,6 $\pm 13,2$ anos, respectivamente, $p=0,0034)$. Porém, não foi observada diferença na média do tempo de espera entre os sexos $(\mathrm{p}=0,229)$. A distribuição dos pacientes por faixa etária mostrou que a média do tempo de espera foi maior no grupo de pacientes que tinha entre 36 e 45 anos $(20,54 \pm 21,21$ meses, $p=0,002)$ quando comparada às demais faixas etárias. A análise dos pacientes por grupo sanguíneo mostrou que a média do tempo de espera foi superior no grupo $\mathrm{O}$ e grupo A quando comparado aos demais grupos $(\mathrm{p}=0,001)$.

Tabela 1: Análise do tempo em lista de espera para a realização de transplante renal de 406 pacientes transplantados entre janeiro de 2018 a dezembro de 2019, no estado do Ceará, em função da faixa etária, sexo e sistema ABO.

\begin{tabular}{|c|c|c|c|}
\hline & $\mathbf{n}(\%)$ & $\begin{array}{l}\text { Tempo médio } \\
\text { de espera (meses) }\end{array}$ & p-Valor \\
\hline Total & 406 & $14.64 \pm 16.18$ & - \\
\hline \multicolumn{4}{|c|}{ Idade $(47 \pm 13 \text { anos; } 18-72)^{\&}$} \\
\hline Até 35 anos & $89(21.9 \%)$ & $11.99 \pm 12.34$ & \multirow[t]{4}{*}{$0,002^{a}$} \\
\hline $36-45$ anos & $95(23.4 \%)$ & $20.54 \pm 21.21^{*}$ & \\
\hline $46-55$ anos & $99(24.4 \%)$ & $13.37 \pm 12.77$ & \\
\hline$>55$ anos & $123(30.3 \%)$ & $13.02 \pm 15.67$ & \\
\hline \multicolumn{4}{|l|}{ Sexo } \\
\hline Feminino & $153(37.7 \%)$ & $13.41 \pm 14.56$ & \multirow[t]{2}{*}{$0,229^{\mathrm{b}}$} \\
\hline Masculino & $253(62.3 \%)$ & $15.38 \pm 17.07$ & \\
\hline \multicolumn{4}{|c|}{ Sistema ABO } \\
\hline A & $142(35.1 \%)$ & $13.23 \pm 13.83^{*}$ & \multirow[t]{4}{*}{$0,001^{a}$} \\
\hline B & $53(13.1 \%)$ & $9.23 \pm 9.07$ & \\
\hline $\mathrm{O}$ & $197(48.6 \%)$ & $17.61 \pm 18.79 *$ & \\
\hline $\mathrm{AB}$ & $13(3.2 \%)$ & $5.77 \pm 7.63$ & \\
\hline
\end{tabular}

*p<0,05 versus demais grupos; ${ }^{\text {TTeste Kruskal-Wallis/Dunn; }{ }^{\mathrm{b}} \text { Teste de Mann-Whitney; }{ }^{\&} \text { média } \pm d e s v i o ~ p a d r a ̃ o, ~ v a l o r e s ~ m i ́ n i m o ~}$ e máximo. Fonte: Autores.

A Tabela 2 evidencia a análise da homozigose e do resultado do PRA em função do tempo de espera para a realização do TxR. Não foi observada influência da presença da homozigose $(\mathrm{p}=0,934)$, do número de homozigoses $(\mathrm{p}=0,448)$ e da homozigose nos diferentes loci HLA (locus $\mathrm{A}, \mathrm{p}=0,169$, locus $\mathrm{B}, \mathrm{p}=0,629$ e locus $\mathrm{DR}, \mathrm{p}=0,064$ ) sobre o tempo de espera.

Não foi observada influência do resultado do PRA no tempo de espera quando se comparou os pacientes que tinham PRA de $0 \%$ com aqueles que apresentavam PRA no intervalo de $1 \% \mathrm{a} \geq 80 \%$ ( $\mathrm{p}=0,790)$. Também não foi observada influência 
quando se estratificou os resultados do PRA em quatro grupos (Tabela 2).

Tabela 2: Análise do tempo em lista de espera para a realização de transplante renal de 406 pacientes transplantados entre janeiro de 2018 a dezembro de 2019, no estado do Ceará, em função da homozigose nos alelos do sistema HLA e dos resultados do PRA.

\begin{tabular}{|c|c|c|c|}
\hline & n (\%) & $\begin{array}{l}\text { Tempo médio } \\
\text { de espera (meses) }\end{array}$ & p-Valor \\
\hline \multicolumn{4}{|l|}{ Homozigose } \\
\hline Não & $328(80,8 \%)$ & $14,78 \pm 16,66$ & $0,934^{\mathrm{a}}$ \\
\hline Sim & $78(19,2 \%)$ & $14,03 \pm 14,05$ & \\
\hline \multicolumn{4}{|c|}{ Quantidade de homozigose } \\
\hline 0 & $328(80,8 \%)$ & $14,78 \pm 16,66$ & $0,448^{\mathrm{b}}$ \\
\hline 1 & $70(17,2 \%)$ & $14,24 \pm 14,30$ & \\
\hline 2 & $6(1,5 \%)$ & $8,67 \pm 11,20$ & \\
\hline 3 & $2(0,5 \%)$ & $22,50 \pm 12,02$ & \\
\hline \multicolumn{4}{|c|}{ Homozigose locus A } \\
\hline Não & $368(90.6 \%)$ & $14.07 \pm 14.65$ & $0,169^{\mathrm{a}}$ \\
\hline $\operatorname{Sim}$ & $38(9.4 \%)$ & $20.11 \pm 26.51$ & \\
\hline \multicolumn{4}{|c|}{ Homozigose locus B } \\
\hline Não & $380(93.6 \%)$ & $14.68 \pm 16.20$ & $0,629^{\mathrm{a}}$ \\
\hline Sim & $26(6.4 \%)$ & $14.00 \pm 16.26$ & \\
\hline \multicolumn{4}{|c|}{ Homozigose locus DR } \\
\hline Não & $382(94.1 \%)$ & $14.25 \pm 15.98$ & $0,064^{a}$ \\
\hline Sim & $24(5.9 \%)$ & $20.79 \pm 18.33$ & \\
\hline \multicolumn{4}{|l|}{ PRA } \\
\hline Não & $311(76.6 \%)$ & $14.39 \pm 15.04$ & $0,790^{\mathrm{a}}$ \\
\hline Sim & $95(23.4 \%)$ & $15.45 \pm 19.52$ & \\
\hline \multicolumn{4}{|l|}{ \%PRA } \\
\hline $0 \%$ & $311(76.6 \%)$ & $14,39 \pm 15,04$ & $0,191^{\mathrm{b}}$ \\
\hline 1 a $49 \%$ & $51(12,6 \%)$ & $15,49 \pm 13,45$ & \\
\hline 50 a $79 \%$ & $20(4,9 \%)$ & $8,80 \pm 10,82$ & \\
\hline $80 \%$ ou mais & $24(5,9 \%)$ & $20,92 \pm 31,58$ & \\
\hline
\end{tabular}

${ }^{*} \mathrm{p}<0,05$ versus demais grupos; ${ }^{\mathrm{a}}$ Teste Mann-Whitney; ${ }^{\mathrm{b}}$ Teste Kruskal-Wallis/Dunn; HLA= antigen leucocyte human; $\% \mathrm{PRA}=$ panel reactive antibodies. Fonte: Autores.

\section{Discussão}

Os resultados do presente estudo sugerem que a homozigose nos alelos A, B e DR do Sistema HLA não influenciou no tempo de espera para a realização do TxR dos 406 pacientes avaliados, mesmo que o paciente apresentasse uma ou mais homozigoses ou que esta ocorresse nos diferentes loci HLA.

Nossos resultados diferem do estudo realizado em São Paulo com 4.230 receptores renais em lista de espera. Os autores mostraram que a frequência de homozigose no alelo DR foi significativamente maior (17\%) do que na população em geral $(11,5 \%, \mathrm{p}<0,0001)$ e que o tempo de permanência em lista de espera dos pacientes com homozigose DR aumentou progressivamente em 12\%,15\%, 21\% e 31\% entre os receptores inscritos na lista há menos de 1 ano (n=996), de 1 a 2 anos $(\mathrm{n}=1649)$, de 3 a 8 anos $(\mathrm{n}=1338)$ e de 9 a 22 anos ( $\mathrm{n}=247)$, respectivamente (DeLima \& Marco, 2013). Acreditamos que esses resultados foram divergentes devido o número de receptores avaliados ter sido cerca de 10 vezes superior ao do presente estudo. Provavelmente, com um número maior de pacientes em homozigose DR fosse possível mostrar uma relação entre a homozigose DR e o tempo de espera, uma vez que o valor de p determinado em 24 pacientes com homozigose DR mostrou uma tendencia a significância $(\mathrm{p}=0,064)$. Além disso, foi relatado o caso de um paciente americano inscrito na lista de espera da CETx do Ceará e que apresenta duas homozigoses, uma no locus B ( $\left.\mathrm{B}^{*} 08, \mathrm{~B} * 08\right)$ e outra no locus DR (DR*03 e DR*03), aguardando o transplante há 99 meses, tempo muito maior do que a média mostrada no presente estudo, tanto na população avaliada ( $\mathrm{n}=406,14,64 \pm 16,18$ meses), quanto nos 78 pacientes com homozigose nos alelos do sistema HLA (20,79 $\pm 18,33$ meses). Da mesma forma, o tempo de espera também foi superior àquele observado nos pacientes com o mesmo grupo sanguíneo que o dele (17,61 \pm 19,79 meses) (Silva, et al., 2019). Em concordância com o estudo realizado em Belo Horizonte, no presente estudo, o tempo de espera na lista para transplante renal foi maior nos pacientes do grupo O (Machado, et al., 2012). De acordo com dois estudos publicados pela Associação Brasileira de Transplante de Órgãos (ABTO 2020a; 2020b), esse fato pode ser explicado pelo maior número de pacientes do grupo O inscritos na CETx do Ceará (62\%). 
A ABTO (2020b) relatou que nos últimos anos, o Brasil esteve em segundo lugar em número absoluto de TxR realizados no mundo, ficando atrás somente dos Estados Unidos. Entre 2015 e 2019, os centros transplantadores do Ceará realizaram 1.259 TxR, ficando em $8^{\circ}$ lugar em relação ao número de transplantes realizados nos 21 estados brasileiros. Em 2019, 6.283 TxR foram realizados em todo Brasil, sendo a grande maioria com rins de doadores falecidos $(\mathrm{n}=5.210,82,9 \%)$. No Ceará, observa-se o mesmo comportamento; dos 293 TxR realizados em 2019, a maioria foi realizado com rim de doador falecido ( $n=272,92,8 \%$ ). Esses achados são fortalecidos pelo fato de o Ceará ser considerado um estado que mais realiza captação de órgãos do Norte e Nordeste. No período avaliado do presente estudo, 463 doadores de órgãos em morte encefálica foram efetivados pela CETx do Ceará e 480 TxR foram realizados a partir desses doadores. Apesar de o Ceará ter alcançado o $3^{\circ}$ lugar em número de doadores falecidos efetivados por milhão da população (28,3 pmp), ficando atrás somente dos estados de Santa Catarina e Paraná, atualmente, ainda restam 757 pacientes com DRC na lista de espera aguardando um rim para transplante (ABTO, 2020b).

Em outro estudo realizado em nosso serviço foi mostrado que a origem étnica do receptor deve ser levada em consideração para se estimar o tempo de espera para realização do transplante, uma vez que determinados alelos HLA são mais frequentes na população do Ceará $(A * 02, A * 24, A * 31, A * 03, B * 15, B * 35, B * 44, B * 51$, DRB1*13, DRB1*04, DRB1*07 e DRB1*15), quando comparado com a frequência alélica de outros estados brasileiros e de outros países (Lima, et al., 2015). Não foi objetivo do estudo determinar a frequência alélica dos 406 pacientes transplantados, mas, provavelmente, esta variável pode estar influenciando o tempo de espera dos 78 pacientes com homozigose nos alelos HLA, uma vez que a Portaria de número 04 de outubro de 2018 do Sistema Nacional de Transplantes não pontua a homozigose na seleção dos potenciais receptores de rins (Brasil, 2017).

Apesar dos resultados do presente estudo não ter demonstrado influência direta da homozigose nos diferentes alelos HLA no tempo de espera dos 406 pacientes transplantados, é importante que o critério de seleção dos receptores seja revisto pelo Sistema Nacional de Transplantes do Ministério da Saúde para que esta condição seja pontuada, pois, somente com essa mudança, pacientes que apresentem alelos HLA raros na população do Ceará tenham chances de transplantar no nosso estado. Sugerimos ainda que possibilitem a inscrição de pacientes que apresentem alelos raros na lista de espera nacional, para que aumentem as possibilidades de encontrar doadores compatíveis.

O resultado do PRA evidencia o grau de sensibilização do paciente frente aos alelos do sistema HLA. Essa sensibilização pode ocorrer em decorrência do número de gestações, transfusões sanguíneas, como também do número de transplantes prévios realizados. Indivíduos altamente sensibilizados, com PRA acima de 80\%, são considerados de difícil transplante, uma vez que o mesmo pode apresentar anticorpos contra os alelos HLA do doador, inviabilizando, na maioria das vezes, a realização do transplante. No presente estudo, o tempo de espera dos pacientes com PRA elevado e homozigose (48,6 $\pm 67,4$ meses) foi superior àquele dos pacientes sem homozigose (15,5 $\pm 17,3$ meses), embora esta diferença não tenha sido significante, provavelmente devido ao pequeno número de pacientes analisados.

\section{Conclusão}

A homozigose nos diferentes alelos do sistema HLA não teve influência significante no tempo em lista de espera para transplante renal dos 406 pacientes inscritos na CETx do Ceará, mesmo naqueles com PRA elevado. Porém, estudos com maior número de pacientes são necessários para melhor avaliar o impacto da homozigose HLA no tempo de espera, em especial no locus DR. Esses resultados serão importantes para se rever os critérios de seleção dos potenciais receptores de rins, uma vez que atualmente a homozigose não é pontuada. 


\section{Referências}

ABTO. (2020a). Dados numéricos da doação de órgãos e transplantes realizados por estado e instituição no período: Janeiro/Setembro - 2020. Registro Brasileiro de Transplantes. 13(3):1-21. https://site.abto.org.br/publicacao/xxvi-no-3-jan-set-de-2020/.

ABTO. (2020b). Dimensionamento dos Transplantes no Brasil e em cada estado (2013-2020). Registro Brasileiro de Transplantes. 16(4),1-89. https://site.abto.org.br/publicacao/xxvi-no-4-anual/.

Brasil. (2014). Diretrizes Clínicas para o Cuidado ao paciente com Doença Renal Crônica - DRC no Sistema Único de Saúde. [Internet]. https://portalarquivos2.saude.gov.br/images/pdf/2014/marco/24/diretriz-cl--nica-drc-versao-final.pdf.

Brasil. (2017). Consolidação das normas sobre os sistemas e os subsistemas do Sistema Único de Saúde. [Internet]. http://portalsinan.saude.gov.br/images/documentos/Legislacoes/Portaria_Consolidacao_4_28_SETEMBRO_2017.pdf.

Crews, D. C., Bello, A. K., \& Saadi, G. (2019). Editorial do Dia Mundial do Rim 2019 - impacto, acesso e disparidades na doença renal. J Bras Nefrol. 41(1):1-9. http://dx.doi.org/10.1590/2175-8239-JBN-2018-0224.

DeLima, M. G., \& Marco, R. (2013). Homozigose no locus HLA-DR dificulta o acesso ao transplante de rim [Internet]. Anais do XIII Congresso Brasileiro de Transplantes. https://site.abto.org.br/wp-content/uploads/2020/06/14-1-1.pdf.

Delves, P. J. (2014). Sistema de antígeno leucocitário humano (HLA) [Internet]. https://www.msdmanuals.com/pt-pt/profissional/imunologiadist\%C3\%BArbios-al\%C3\%A9rgicos/biologia-do-sistema-imunit\%C3\%A1rio/sistema-de-ant\%C3\%ADgeno-leucocit\%C3\%A1rio-humano-hla.

Despande, A. (2017). The Human Leukocyte Antigen System...Simplified. Global Journal of Transfusion Medicine. 2(2):77-88. http://dx.doi.org/10.4103/GJTM.GJTM_45_17.

Garcia, C. D., Pereira, J. D., \& Garcia, V. D. (2015). Doação e transplante de órgãos e tecidos. Libbs.

Goldberg, A. C., \& Rizzo, L. V. (2015). Estrutura do MHC e função: apresentação de antígenos. Einstein. 1(13):153-156. http://dx.doi.org/10.1590/S1679$45082015 \mathrm{RB} 3123$.

Lima, M. S., Coelho, R. V. M., \& Silva, S. F. R. (2015). Frequência alélica do sistema HLA em doadores de órgãos em morte encefálica do Ceará [trabalho de conclusão de curso, Universidade de Fortaleza].

Machado, E L., Gomes, I. C., Acurcio, F. A., César, C. C., Almeida, M. C. M., \& Cherchiglia, M. L. (2012). Fatores associados ao tempo de espera e ao acesso ao transplante renal em Belo Horizonte, Minas Gerais, Brasil. Cad. Saúde Pública. 28(12):2315-2326. https://doi.org/10.1590/S0102$311 \mathrm{X} 2012001400010$.

Marinho, A. W. G. B., Penha A. P., Silva, M. T., \& Galvão, T. F. (2017). Prevalência de doença renal crônica em adultos no Brasil: revisão sistemática da literatura. Cad. Saúde Colet. 25(3):379-388. https://doi.org/10.1590/1414-462X201700030134.

Pécora RAA. (2016). Associação dos anticorpos anti-HLA pré-formados e da compatibilidade HLA à rejeição celular aguda precoce no transplante hepático [tese de doutorado, USP]. https://doi.org/10.11606/T.5.2016.tde-09082016-154954.

Romão Jr, J. E. (2004). Doença renal crônica: definição, epidemiologia e classificação. J Bras Nefrol. 26(3):1-3. https://www.bjnephrology.org/article/doencarenal-cronica-definicao-epidemiologia-e-classificacao/.

Santos, B. P., Viegas, A. C., Paula, E. A., Lise, F., Rodrigues, L. P. V., Fuculo Junior, P. R. B., \& Schwartz, E. (2018). Percepção de pessoas submetidas ao transplante renal sobre a doação de órgãos. ABCS health sci. 43(1):30-35. https://doi.org/10.7322/abcshs.v43i1.928.

Santos, L.C. (2011). Polimorfismos do gene HLA-E em uma amostra de afroparanaenses doadores de medula óssea. [dissertação, Universidade Federal do Paraná]. https://acervodigital.ufpr.br/handle/1884/26828?show=full.

Silva, S. F. R., Ribeiro, I. F., Almeida, E. R. B., Félix, M. E. C., Ávila, A. V. M., \& Silva, S. L. (2019). Perspectiva de transplante com rim de doador falecido de paciente com alelos HLA incomuns: $\mathrm{O}$ que fazer? [Internet]. Anais do XVI Congresso Brasileiro de Transplante. https://sistemaparaevento.com.br/evento/abto2019/trabalhosaprovados/naintegra/13153.

SBN. (2019). Tratamentos [Internet]. https:// sbn.org.br/orientações-e-tratamentos/tratamentos. 